\title{
Two Universal Extra Dimensions
}

\author{
Gustavo Burdman \\ Instituto de Física, Universidade de São Paulo \\ R. do Matão, Travessa R, 187, Cidade Universitaria \\ São Paulo, SP 05580-900, Brazil
}

\begin{abstract}
We review the main results of the study of the Standard model propagating in two universal extra dimensions. Gauge bosons give rise to heavy spin- 1 and spin-0 particles. The latter constitute the lightest Kaluza-Klein $(\mathrm{KK})$ particle in the level $(1,0)$. The level $(1,1)$ can be s-channel produced. The main signals at the Tevatron and the LHC will be several $t \bar{t}$ resonances.
\end{abstract}

\section{INTRODUCTION}

If compact universal extra dimensions (UED) exist their compactification radius can be as large as few hundred $\mathrm{GeV}^{-1}$ [1]. This is due to the restrictions imposed by $\mathrm{KK}$ parity.

The degenerate KK spectrum is split by radiative corrections [3]. However, these splittings are generically small and make the phenomenology at hadron colliders rather nontrivial [2] due to the relatively small energy release in the decay chains. Alternatively, level 2 KK modes can be s-channel produced [4]. Their couplings to zero modes are suppressed by volume (or loop) factors, but since they can decay through these couplings to light particles, they release enough energy. On the other hand, in the 5D case, the mass of the 2 nd KK level is just $2 / R$, twice the first level, up to radiative corrections. Then, these excitations in principle can also decay to two level $1 \mathrm{KK}$ modes, so that the clean decays of the 2 nd KK level to two zero modes have to compete with these not-so-clean channels.

\section{TWO UNIVERSAL EXTRA DIMENSIONS}

Considering the case with two Universal Extra Dimensions (TUED) has several motivations. It is possible to protect the proton from decaying [5], and requiring the cancellation of chiral anomalies implies the existence of three families. However, here we concentrate on the phenomenological differences with the case of one UED.

There are two main aspects that differentiate TUED theories from the one UED case. First, the level-2 vector modes in the TUED offer better opportunities for discovery since they have masses which are larger than the level-1 masses by a factor of approximately $\sqrt{2}$. As a result, their production is possible at smaller center-of-mass energies, and the decays of level-2 states into pairs of level-1 states, which would lead to only soft leptons and jets in the detector, are kinematically forbidden (as opposed to the case of one UED where such decays are typically allowed). Then the level- 2 states, characterized by KK numbers $(1,1)$, have large branching fractions for decays into a pair of standard model 
particles giving rise to a high $p_{T}$ signal. The second distinctive feature of TUED is that each vector mode is accompanied by a spin- 0 particle in the adjoint representation of the corresponding gauge group. These are the un-eaten Nambu-Goldstone bosons of the breaking of translation invariance. These two features will drive the phenomenology of the TUED to be quite distinctive.

We compactify the TUED on a chiral square as defined in Refs. [7]. It consists of a square, $0 \leq x_{4}, x_{5} \leq \pi R$, where $x_{4}, x_{5}$ are the coordinates of the extra dimensions and $R$ is the compactification "radius". The compactification is obtained by imposing the identification of two pairs of adjacent sides of the square. The KK modes are characterized by two numbers, $j$ and $k$, integers with $j \geq 1$ and $k \geq 0$, or $j=k=0$. The 4D fields $\Phi^{(j, k)}\left(x^{\mu}\right)$ are the KK modes of the 6D field $\Phi(x, y)$. They have masses due to the momentum along $x^{4}, x^{5}$ given by $M_{j, k}=\frac{1}{R} \sqrt{j^{2}+k^{2}}$, so that the mass spectrum, in the limit where other contributions to physical masses are neglected, starts with $M_{0,0}=0$, $M_{1,0}=1 / R, M_{1,1}=\sqrt{2} / R, M_{2,0}=2 / R, \ldots$

The 6D gauge bosons decompose into a tower of 4D spin-1 fields, a tower of 4D spin- 0 fields that are eaten by the spin- 1 states, plus a tower of spin- 0 fields that remain in the spectrum. So there will be adjoint scalars: $G_{H}^{a(j, k)}$ corresponding to the $6 \mathrm{D}$ gluon field, and $W_{H}^{ \pm(j, k)}, W_{H}^{3(j, k)}$ and $B^{(j, k)}$, corresponding the the electroweak 6D fields. This is a crucial difference with the 5D case. In what follows we summarize the results of Ref. [8], where a detailed discussion on the KK spectrum and couplings, production cross sections and branching ratios can be found.

\subsection{Localized Operators}

The compactification on the chiral square implies the presence of three singularities. Operators localized on these fixed points are generated at one loop by the bulk interactions, as well as by ultra-violet physics. We demand that the UV contributions respect KK parity so as to keep the possibility of having a dark matter candidate. Then we have

$$
\begin{aligned}
& \int_{0}^{\pi R} d x^{4} \int_{0}^{\pi R} d x^{5}\left\{\mathscr{L}_{\text {bulk }}+\delta\left(x_{4}\right) \delta\left(\pi R-x_{5}\right) \mathscr{L}_{2}\right. \\
& \left.+\left[\delta\left(x_{4}\right) \delta\left(x_{5}\right)+\delta\left(\pi R-x_{4}\right) \delta\left(\pi R-x_{5}\right)\right] \mathscr{L}_{1}\right\} .
\end{aligned}
$$

The operators in $\mathscr{L}_{1}$ and $\mathscr{L}_{2}$ will give rise to KK number violating (but KK-parity conserving) contributions to the KK masses and couplings. For instance, for gauge kinetic term we have

$$
-\frac{1}{4} \frac{C_{p G}}{\Lambda^{2}} G^{\mu v} G_{\mu v}-\frac{1}{2} \frac{C_{p G}^{\prime}}{\Lambda^{2}}\left(G_{45}\right)^{2}
$$

with $p=1,2$, and similarly for fermion kinetic terms. Naive dimensional analysis can be used to estimate that the coefficients of these operators are of order $C \sim \ell_{6} / \ell_{4} \simeq 8 \pi$, where $\ell_{6}$ and $\ell_{4}$ are loop factors in $6 \mathrm{D}$ and $4 \mathrm{D}$ respectively. On the other hand, the cutoff satisfies $\Lambda R \simeq\left(32 /\left(\alpha_{s} N_{c}\right) \simeq 10\right.$. This leads to the NDA estimate of $\sim g^{2} / 16 \pi^{2}$ for the effective $4 \mathrm{D}$ couplings induced by the $C$ 's. 
Bulk interactions at one loop renormalize the localized operators below $\Lambda$, generating large logarithms. The logarithmic enhancement, approximately $\ln (\Lambda R) \simeq 2.3$, is not particularly large. However, in order to estimate the KK spectrum and the KK-number violating couplings we will make use of these calculable contributions.

For instance, we can compute the one-loop contributions to the KK mass spectrum. One important finding is the fact that the lightest KK particle (LKP) of the first level (the level $(1,0)$ ), is the scalar adjoint corresponding the the $U(1)_{Y}$ gauge boson, $B_{H}$. The rest of the spectrum for the first and second KK level can be seen in detail in Ref. [8].

\subsection{KK-Number-Violating Interactions}

These interactions are generated by the localized operators. For instance, the interactions of two zero-mode quarks with KK gluons is given by

$$
g_{s} C_{j, k}^{q G}\left(\bar{q} \gamma^{\mu} T^{a} q\right) G_{\mu}^{(j, k) a}
$$

where $g_{s}$ is the QCD coupling and, as we mentioned earlier, the coefficients such as $C_{j, k}^{q G}$ receive contributions from above $\Lambda$ as well as logarithmically enhanced contributions below the cutoff, which result in $\left(g^{2} N_{c} / 16 \pi^{2}\right) \ln \left(M_{s}^{2} / \mu^{2}\right)$. The typical size of these couplings are then this loop factor time log, times a coefficient of order one, which can be estimated from the bulk one-loop contributions.

The KK spinless adjoints interact with the zero-mode quarks only via dimension- 5 or higher operators:

$$
\frac{g_{s} \tilde{C}_{j, k}^{q G}}{M_{j, k}}\left(\bar{q} \gamma^{\mu} T^{a} q\right) D_{\mu} G_{H}^{(j, k) a}
$$

where $\tilde{C}_{j, k}^{q G}$ are real dimensionless parameters, and $D_{\mu}$ is the gauge covariant derivative. It is important to notice that the vertex (2.4) is proportional to the quark masses, as can be seen by integrating by parts and using the fermion equations of motion. As a result, the spinless adjoints decay almost exclusively into top quarks. This observation also implies that the coupling for direct production of the spinless states is negligible, being suppressed by the $u$ or $d$-quark masses. However, the spinless adjoints can be easily produced in the decays of KK quarks or leptons.

\section{PHENOMENOLOGY OF THE 6D STANDARD MODEL}

The first KK level $(1,0)$ must be pair produced. On the other hand, the second KK level, $(1,1)$, can be produced in the $s$ channel through KK-number violating interactions such as (2.3) or (2.4). If we assume that the KK-number violating couplings are mostly radiatively induced, then the corresponding couplings of the electroweak level-2 KK modes to zero-mode leptons is suppressed with respect to the ones with zero-mode quarks: the electroweak level-2 KK modes are therefore mostly leptophobic. Although the level-2 KK gluon $G_{\mu}^{(1,1)}$ has a larger production cross section than the electroweak 
gauge bosons, its mass is also larger (is radiatively enhanced). Then, the number of events generated through level-2 KK gluons is comparable to the one originating from the cascade decays of electroweak level-2 KK gauge bosons.

The fact that the dominant decay channels are hadronic, even for the electroweak KK modes, implies that the best hope for observation is in the $t \bar{t}$ channel. This is particularly true due to the fact that a significant fraction of the produced level-2 KK modes results in adjoint scalars, which decay almost exclusively into $t \bar{t}$ pairs. The five resonances that decay to $t \bar{t}$, will form three distinguishable peaks: $G_{H}^{(1,1)}+W_{\mu}^{3(1,1)}$, with $\sim 1.10 M_{1,1}$; $B_{\mu}^{(1,1)}+W_{H}^{3(1,1)}$, with $\sim 0.97 M_{1,1}$; and $B_{H}^{(1,1)}$, with $\sim 0.86 M_{1,1}$, where $M_{(1,1)}=\sqrt{2} / R$ is the level-2 un-corrected KK mass. In Ref. [8] the reach of the Tevatron was studied. It was found that the Tevatron can observe level- $2 \mathrm{KK}$ modes in the $t \bar{t}$ channel up to about $700 \mathrm{GeV}$. The ultimate Tevatron reach for the compactification scale is found to e about $R^{-1} \sim 500 \mathrm{TeV}$. The production cross sections at the LHC were estimated using $q \bar{q}$ initial states only. It was found that the LHC should have a considerably larger reach on $R^{-1}$. However, more detailed work is needed to include all contributions as well as a serious study of the backgrounds.

Finally, a comment about the viability of the SM in UED and TUED. It was recently pointed in Ref. [9] that the SM in 5D UED has a "little hierarchy" problem since the Higgs mass still receives quadratically divergent contributions. Solving this problem in the 5D scenario by assuming the Higgs is a pseudo-Nambu-Goldstone boson, results in the cutoff dependence becoming linear. The phenomenology might change with respect to the SM in 5D depending on the mechanism used to solve the little hierarchy. However, in the 6D case of TUED, the cutoff dependence remains quadratic. Then more work is needed to address the stabilization of the Higgs mass in a theory with two inversal extra dimensions.

\section{REFERENCES}

1. T. Appelquist, H. C. Cheng and B. A. Dobrescu, Bounds on universal extra dimensions Phys. Rev. D 64, 035002 (2001) [arXiv:hep-ph/0012100].

2. H. C. Cheng, K. T. Matchev and M. Schmaltz, Bosonic supersymmetry? Getting fooled at the LHC, Phys. Rev. D 66, 056006 (2002) [arXiv:hep-ph/0205314].

3. H. C. Cheng, K. T. Matchev and M. Schmaltz, Radiative corrections to Kaluza-Klein masses, Phys. Rev. D 66, 036005 (2002) [arXiv:hep-ph/0204342].

4. A. Datta, K. Kong and K. T. Matchev, Discrimination of supersymmetry and universal extra dimensions at hadron colliders, [arXiv:hep-ph/0509246].

5. T. Appelquist, B. A. Dobrescu, E. Ponton and H. U. Yee, Proton stability in six dimensions, Phys. Rev. Lett. 87, 181802 (2001) [arXiv:hep-ph/0107056].

6. B. A. Dobrescu and E. Poppitz, Number of fermion generations derived from anomaly cancellation, Phys. Rev. Lett. 87, 031801 (2001) [arXiv:hep-ph/0102010].

7. B. A. Dobrescu and E. Pontón, Chiral compactification on a square, JHEP 0403, 071 (2004) [arXiv:hep-th/0401032];

G. Burdman, B. A. Dobrescu and E. Ponton, Six-Dimensional Gauge Theory On The Chiral Square, JHEP 0602, 033 (2006) |arXiv:hep-ph/0506334|.

8. G. Burdman, B. A. Dobrescu and E. Ponton, Resonances from two universal extra dimensions, Phys. Rev. D 74, 075008 (2006) [arXiv:hep-ph/0601186].

9. G. Burdman and A. G. Dias, The little hierarchy in universal extra dimensions, arXiv:hep-ph/0609181]. 